\title{
DETERMINAÇÃO DOS PARÂMETROS CINÉTICOS E TERMODINÂMICOS DA ADSORÇÃO DE L-CISTEÍNA EM OURO POR MEIO DA TÉCNICA DE MICROBALANÇA A CRISTAL DE QUARTZO
}

\author{
Adriano dos Santos, Josiel José da Silva e Paulo Roberto Bueno* \\ Instituto de Química, Universidade Estadual Paulista, Rua Francisco Degni, 55, 14800-900 Araraquara - SP, Brasil \\ Ailton Massaiti Watanabe \\ Faculdade de Filosofia, Ciências e Letras de Ribeirão Preto, Universidade de São Paulo, Av. Bandeirantes, 3900, 14040-901 \\ Ribeirão Preto - SP, Brasil
}

Recebido em 26/9/11; aceito em 9/2/12; publicado na web em 15/6/12

\begin{abstract}
DETERMINATION OF KINETIC AND THERMODYNAMIC PARAMETERS OF L-CYSTEINE ADSORPTION ONTO GOLD BY THE QCM TECHNIQUE. This article discusses the adsorption kinetics of a L-cysteine monolayer onto a gold surface by means of information obtained through the QCM technique. The results indicate that the adsorption process is rapid and follows the Langmuir isotherm, in which adsorption and desorption are considered. From these measurements the following parameter values were obtained: $k_{d}=(4.2 \pm 0.4) \times 10^{-3} \mathrm{~s}^{-1}, k_{a}=75 \pm 6 \mathrm{M}^{-1} \mathrm{~s}^{-1}, K_{e q}=(1.8 \pm 0.3) \times 10^{4} \mathrm{M}^{-1}$ and $\Delta G_{a d s}=-(5.8 \pm 0.2) \mathrm{kcal} \mathrm{mol}^{-1}$.
\end{abstract}

Keywords: L-cysteine; QCM; SAM.

\section{INTRODUÇÃO}

Tióis (moléculas que possuem o grupo -SH) são substâncias orgânicas que se adsorvem espontaneamente em substratos metálicos como ouro, prata e paládio, formando monocamadas estruturalmente organizadas. ${ }^{1-5}$ Essas nanoestruturas, conhecidas como SelfAssembled Monolayers (SAMs), conferem interessantes propriedades físicas e químicas a superfícies, dependendo da molécula de tiol utilizada. ${ }^{3}$ Dentre essas aplicações, pode-se citar sistemas para prevenção de corrosão, catálise, sensores químicos ${ }^{3}$ e camada adesiva para imobilização de biomoléculas na construção de biossensores. ${ }^{6-8}$

Estudos sobre a cinética de adsorção de alcanotióis a partir de soluções diluídas $\left(10^{-3} \mathrm{M}\right)$ em superfície de $\mathrm{Au}(111)$ sugerem que esse processo ocorre em duas etapas: ${ }^{9}$ a primeira muito rápida, durando de segundos a minutos, em que ocorre o processo de adsorção química do tiol no ouro formando tiolato (explicada pela isoterma de adsorção de Langmuir ${ }^{9,10}$ ) e a segunda, durando de horas a dias, em que ocorre o processo de organização da camada formada. ${ }^{9}$ A cinética da primeira etapa está relacionada com a interação entre a superfície metálica e o átomo de enxofre do grupo tiol, enquanto a da segunda está relacionada com a desordem entre as cadeias das moléculas, com os diferentes componentes das interações entre cadeia-cadeia (interações de van der Waals, dipolo-dipolo, ligação de hidrogênio) e com a mobilidade das cadeias moleculares do adsorbato na superfície do metal. ${ }^{9}$

Mccarley et al. ${ }^{11}$ ao estudarem a formação de monocamadas de tióis por STM, notaram que a ligação entre o ouro e a molécula de tiol é lábil, ou seja, capaz de se movimentar entre dois sítios de adsorção adjacentes. ${ }^{3}$ Esse fato sugere que o mecanismo de formação de monocamada de tiol explicado por dois estágios não mostra uma imagem completa do processo, e que mecanismos de adsorção/ dessorção podem ser relevantes na formação da estrutura da monocamada, bem como no processo de adsorção do tiol em ouro. Esse fato também foi observado por Karpovich e Blanchard, ${ }^{12}$ utilizando a técnica de microbalança a cristal de quartzo, QCM (Quartz Crystal Microbalance), ao estudarem a cinética de adsorção de alcanotióis de cadeia longa ( $\mathrm{HS}-\left(\mathrm{CH}_{2}\right)_{\mathrm{n}}-\mathrm{CH}_{3}, n=7$ e 17) em ouro.

Embora existam evidências de um complexo processo de formação de monocamadas de tióis, poucos estudos se dedicaram à análise in situ da formação desses filmes, ${ }^{13}$ sendo que na grande maioria, pesquisas exploraram a caracterização desses filmes após sua formação, ${ }^{5,9,14}$ o que não permite obter informações a respeito de processos de adsorção e dessorção durante a formação dessas estruturas.

Uma molécula amplamente utilizada na construção de biossensores é a cisteína. ${ }^{15-18}$ Esse aminoácido é comumente empregado na obtenção de monocamadas automontadas, via ligação do grupo tiol sobre superfície de ouro, ${ }^{16,19-21}$ que tem sido caracterizada por várias técnicas. ${ }^{15,19}$ Entretanto, nenhum estudo abordou os mecanismos de formação in situ desses filmes com relação a processos de adsorção e dessorção.

Com o intuito de analisar a cinética de adsorção da L-cisteína em ouro e verificar a existência de processos de dessorção, foram realizados experimentos in situ utilizando a técnica de QCM. Por meio dela, é possível medir a taxa de adsorção de moléculas de tiol na superfície de ouro em tempo real e estimar as constantes cinéticas de adsorção $\left(k_{a}\right)$ e dessorção $\left(k_{d}\right)$, bem como a constante de equilíbrio da reação $\left(K_{e q}\right)$ e a energia livre de adsorção $\left(\Delta \mathrm{G}_{a d s}\right)$.

A QCM é uma técnica fundamentada na propriedade piezelétrica do cristal de quartzo. ${ }^{22-24}$ Esse material pode ser posto a oscilar através da aplicação de uma diferença de potencial alternada, sendo que sua frequência de oscilação é monitorada em função do tempo. Por meio da relação de Sauerbrey ${ }^{24,25}$ (Equação 1) é possível relacionar a variação de frequência de oscilação do cristal com a massa aderida em sua superfície.

$$
\Delta f^{\prime}=-\frac{f_{0}^{2}}{F_{q} \rho_{q} A_{e l}} \Delta m
$$

onde $\Delta f$ ' é a variação da frequência devido ao recobrimento do eletrodo, $f_{0}$ a frequência fundamental do cristal, $\Delta m$ a massa de material depositado, $\rho_{q}$ a densidade do cristal, $F_{q}$ é a constante de frequência ( $F_{q}=f_{0} d_{q}$, onde $d_{q}$ é a espessura do cristal) e $A_{e l}$ é a área do eletrodo sobre o cristal. Essa equação pode ser reescrita de forma simplificada (Equação 2):

$$
\Delta f^{\prime}=-n \frac{1}{C} \Delta m
$$


sendo que $n$ é o número do harmônico $(n=1,3,5,7 \ldots)$ e $C$ o coeficiente de sensibilidade teórica da QCM $\left(17,7 \mathrm{ng} \mathrm{Hz}^{-1} \mathrm{~cm}^{-2}\right.$ para cristal de $5 \mathrm{MHz}$ ). O sinal negativo da equação evidencia que um aumento de massa sobre o cristal acarreta um decréscimo de sua frequência de oscilação.

No caso em que a massa aderida não for rígida, significa que seu movimento não está acoplado totalmente ao movimento oscilatório do cristal, causando perdas energéticas. Nesses casos, apenas os valores de frequência não são adequados para estimar a massa aderida sobre o substrato e se faz necessário conhecer os valores de dissipação energética (fator $D$ ), fornecendo informações a respeito da viscoelasticidade da camada formada. ${ }^{24}$

De forma geral, quanto maior for a viscoelasticidade da camada formada, maior será o amortecimento e, portanto, o valor de $D$. Esse parâmetro é definido como ${ }^{24}$ (Equação 3):

$$
D=\frac{E_{\text {dissipada }}}{2 \pi E_{\text {armazenada }}}
$$

sendo $E_{\text {dissipada }}$ a energia dissipada por ciclo de oscilação do cristal e $E_{\text {armazenada }}$ a energia total armazenada no sistema oscilatório.

Por meio do monitoramento simultâneo de $\Delta f(t)$ e $\Delta D(t)$ é possível estudar a formação de monocamadas de tióis e inferir suas características viscoelásticas.

\section{PARTE EXPERIMENTAL}

O aminoácido L-cisteína (Sigma) foi utilizado sem prévia purificação. As soluções aquosas foram preparadas em água deionizada.

$\mathrm{O}$ estudo cinético da formação das monocamadas foi realizado utilizando a técnica de microbalança a cristal de quartzo (Q-Sense modelo E4). A técnica de QMC-D permite obter os valores de $\Delta f$ e $\Delta D$ durante o processo de formação da monocamada em função do tempo.

Os cristais de quartzo utilizados (QSX-301, especificações: corte AT, frequência fundamental de $4,95 \mathrm{MHz} \pm 50 \mathrm{KHz}, 14 \mathrm{~mm}$ de diâmetro e $0,3 \mathrm{~mm}$ de espessura) são recobertos com fina camada de ouro em ambos os lados.

Na montagem da célula (modelo QFM 401), os cristais de quartzo são dispostos de forma que uma de suas faces fica em contato com a solução de tiol sendo investigada (capacidade de volume sobre o cristal de quartzo $\approx 40 \mu \mathrm{L}$ ). A célula permite controle de temperatura de $\pm 0,5^{\circ} \mathrm{C}$. Para a injeção das soluções de tiol foi utilizada uma bomba peristáltica Ismatec - modelo IPCN de quatro canais.

Os cristais foram limpos em solução piranha $\left(\mathrm{H}_{2} \mathrm{SO}_{4}\right.$ (concentrado): $\mathrm{H}_{2} \mathrm{O}_{2} ; 7: 3, \mathrm{v} / \mathrm{v}$ ) previamente preparada, lavados exaustivamente com água deionizada e acetona e secos em nitrogênio. Os cristais foram utilizados imediatamente após a limpeza.

Para a realização dos ensaios, o terceiro e quinto harmônicos de oscilação do cristal de quartzo $\left(\Delta f_{\mathrm{n}=3} / 3\right.$ e $\left.\Delta f_{\mathrm{n}=5} / 5\right)$ e os respectivos parâmetros de dissipação $\left(\Delta D_{\mathrm{n}=3}\right.$ e $\left.\Delta D_{\mathrm{n}=5}\right)$ foram estabilizados injetando-se água deionizada em fluxo contínuo $(1,75 \mu \mathrm{L} / \mathrm{s})$ por aproximadamente $10 \mathrm{~min}$. Todas as medidas apresentadas nesse trabalho correspondem ao terceiro harmônico. Para cada ensaio, injetou-se solução aquosa de L-cisteína $\left(0,1 \times 10^{-4} \mathrm{M}, 0,5 \times 10^{-4} \mathrm{M}, 1,0 \times 10^{-4} \mathrm{M}, 2,5 \times 10^{-4} \mathrm{M} \mathrm{e} \mathrm{5,0}\right.$ x $10^{-4} \mathrm{M}$ ) recém-preparada em fluxo de $1,75 \mu \mathrm{L} / \mathrm{s}$ por $30 \mathrm{~min}$, tempo suficiente para restabelecer a estabilidade da frequência e garantir que todo o volume da célula fosse preenchido com a solução. Após a injeção da solução, o fluxo foi desligado e a solução ficou em contato com o cristal por mais $40 \mathrm{~min}$. Após esse tempo, o fluxo de água deionizada foi novamente ligado, objetivando retirar moléculas de aminoácido não adsorvidas. Não houve alteração significativa do pH das soluções após o processo de adsorção. Os ensaios foram realizados em temperatura ambiente $\left(25^{\circ} \mathrm{C}\right)$ e em triplicata.

\section{RESULTADOS E DISCUSSÃO}

São mostrados, na Figura 1, os gráficos do terceiro harmônico de oscilação em função do tempo para as diferentes concentrações de L-cisteína utilizadas.
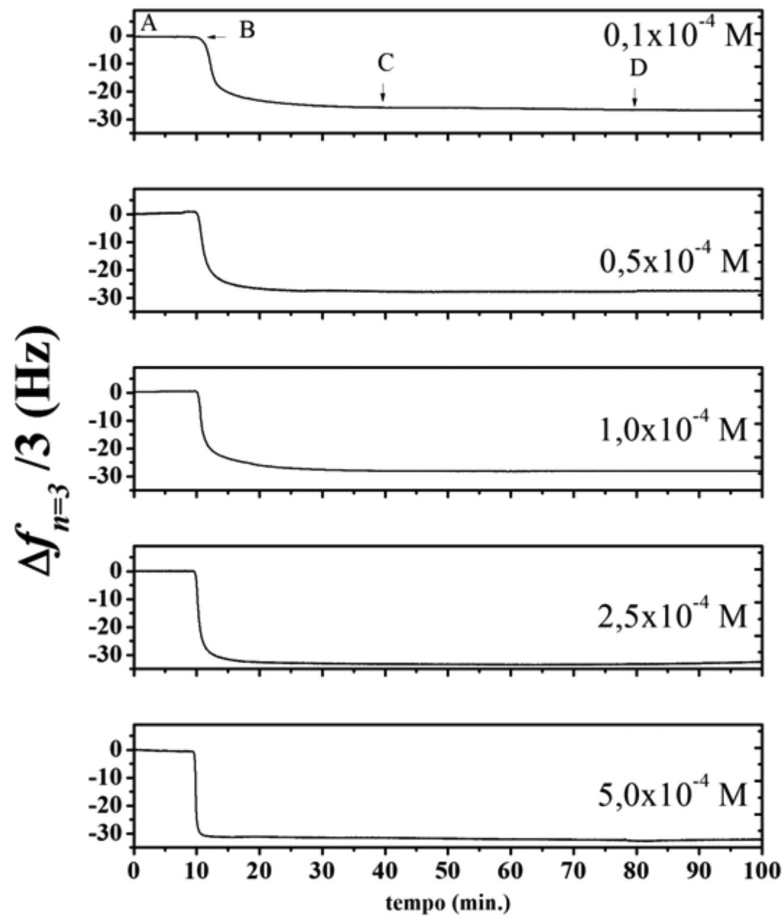

Figura 1. Variação da frequência de oscilação $\left(\Delta f_{\mathrm{n}=3} / 3\right)$ em função do tempo para a formação das monocamadas de L-cisteína em diferentes concentrações. $O$ intervalo A-B corresponde à etapa de estabilização injetando-se água deionizada por $10 \mathrm{~min}$. A seta em $B$ representa o início da injeção de solução de aminoácido, que é parada em C. A partir de D, o fluxo de água deionizada é novamente ligado

O intervalo de A a B corresponde ao período de estabilização da frequência de oscilação do cristal de quartzo por um período de 10 min. A partir do ponto $\mathrm{B}$ (indicado pela seta à esquerda) é iniciada $\mathrm{a}$ injeção da solução de L-cisteína. É possível observar uma queda de $\Delta f$ em todas as concentrações até um valor final constante. Pela relação de Sauerbrey (Equação 1), é possível afirmar que o aminoácido está se adsorvendo na superfície de ouro, visto que a variação negativa de $\Delta f$ corresponde a um acúmulo de massa ( $\Delta m$ positivo) na superfície do ouro. $\mathrm{O}$ ponto $\mathrm{C}$ (indicado pela seta à direita) corresponde ao final da injeção da amostra, enquanto a seta em $\mathrm{D}$ indica o início da injeção de água deionizada, não ocorrendo alteração nos valores de $\Delta f$.

Levando em consideração que o processo de ligação do tiol no ouro finaliza quando não há mais adsorção aparente, ou seja, quando $\Delta f$ permanece constante, é possível afirmar que a adsorção de L-cisteína nas concentrações estudadas é alcançada em tempo na ordem de minutos, em concordância com o trabalho de Qingwen et al. ${ }^{19}$ Além do mais, a rapidez em que esse processo é alcançado, ou seja, o equilíbrio químico de adsorção do tiol no ouro, depende da concentração de aminoácido utilizada, e essa informação é a chave para determinar os parâmetros cinéticos e termodinâmicos da adsorção do tiol. ${ }^{12}$

O mecanismo de adsorção de tiol no ouro ainda não é muito claro. ${ }^{3}$ Entretanto, sugere-se ${ }^{12}$ que a adsorção de tiol, formando tiolato (Au$\mathrm{SR}$ ), é um equilíbrio químico, ocorrendo desprotonação da molécula de tiol seguida da eliminação de molécula $\mathrm{H}_{2}$ da superfície do metal:

$$
\mathrm{Au}_{\text {(s) }}+\mathrm{RS}-\mathrm{H}_{\text {(solv. })} \rightleftarrows \mathrm{RS}-\mathrm{Au}_{\text {(s) }}+1 / 2 \mathrm{H}_{2 \text { (solv. })}
$$


A energia de adsorção calculada a partir de valores teóricos ${ }^{9}$ $\left(-5 \mathrm{Kcal} \mathrm{mol}^{-1}\right)$ está em concordância com trabalhos experimentais encontrados na literatura, ${ }^{10}$ desconsiderando as interações intermoleculares entre as cadeias das moléculas adsorvidas no substrato.

Vários estudos indicam que a isoterma de Langmuir pode ser utilizada para descrever a cinética de adsorção do tiol em ouro. ${ }^{9,12}$ Esse modelo assume que há apenas a formação de uma monocamada, que toda a superfície é equivalente e que a adsorção de uma molécula em um sítio independe se há ou não um sítio vizinho ocupado; em outras palavras, estes sítios podem ser considerados quimicamente semelhantes. Com relação à primeira exigência, esta é válida para o estudo de formação de camadas de tióis sobre superfície de ouro, visto que essas moléculas formam monocamadas sobre essa superfície. ${ }^{5} \mathrm{~A}$ segunda hipótese, entretanto, considera que a superfície esteja livre de defeitos e rugosidade. Certamente, essa hipótese não pode ser considerada fisicamente realista, embora estudos demonstrem que essa condição não restrinja o uso do modelo. ${ }^{12}$ A terceira hipótese assume que as moléculas de tiol não interagem com aquelas localizadas em sítio vizinho ocupado. Essas interações se tornam relevantes em condições de saturação de ocupação dos sítios, ou seja, quando a superfície se encontra totalmente coberta pela monocamada. ${ }^{12}$ Além do mais, a escala de tempo associada ao processo de interação entre essas moléculas é muito maior do que aquela associada ao processo de adsorção, ${ }^{12}$ ou seja, quando o tempo relacionado ao processo adsortivo está compreendido na ordem de segundos a minutos, o processo organizacional, que corresponde à etapa de interações intermoleculares, está na ordem de horas a dias. Consequentemente, os efeitos do processo organizacional da monocamada podem ser desprezados devido à escala de tempo utilizada para o estudo do processo de adsorção de L-cisteína no ouro, que foi da ordem de minutos. Importante notar também que a isoterma de Langmuir foi desenvolvida para processos de adsorção física, mas como a energia de ligação associada à adsorção de tiol em ouro é suficientemente pequena, essa condição não interfere seriamente na aplicação do modelo, conforme afirmação de Karpovich. ${ }^{12}$

A equação que relaciona a taxa de adsorção é dada pela seguinte relação ${ }^{12}$ (Equação 4):

$$
\frac{d \theta}{d t}=k_{a}(1-\theta) C-k_{d} \theta
$$

sendo $\theta$ a fração da superfície recoberta, $(1-\theta)$ a fração da superfície exposta, $C$ a concentração do tiol, e $k_{a}$ e $k_{d}$ as constantes de associação e dissociação, respectivamente. Por meio da integração dessa equação, obtém-se a Equação 5:

$$
\theta(t)=\frac{C}{C+\left(k_{d} / k_{a}\right)}\left[1-e^{\left(-\left(k_{a} C+k_{d}\right) t\right)}\right]
$$

Esse resultado pode ser simplificado considerando que $\tau=k_{d}+$ $k_{a} C$ e $K^{\prime}=C /\left(C+\left(k_{d} / k_{a}\right)\right)$, fornecendo a relação (Equação 6):

$$
\theta(t)=K^{\prime}\left[1-e^{(-\tau t)}\right]
$$

O parâmetro $\tau$ é conhecido como constante de relaxação. $K^{\prime}$ representa a máxima cobertura de adsorbato em ouro numa dada concentração no equilíbrio.

Um aumento do grau de cobertura no tempo, $\theta(t)$, corresponde a um aumento de massa sobre o sensor, sendo o seu valor proporcional a $\Delta m$. Portanto, a relação pode ser expressa em termos de massa adsorvida sobre a superfície do cristal (Equação 7):

$$
\Delta m(t)=\Delta m_{\operatorname{máx}}\left[1-e^{(-\tau t)}\right]
$$

A Equação de Sauerbrey pode ser aplicada a sistemas líquidos desde que a massa aderida na superfície do cristal de quartzo seja rígida e uniforme em toda a extensão da superfície. Uma forma de evidenciar a formação de filme com essas características é por meio da análise dos harmônicos de frequência de oscilação do cristal, bem como pelo respectivo parâmetro de dissipação, $\Delta D$. Se os harmônicos se sobrepuserem e $\Delta D$ assumir baixos valores em relação à frequência, $-\Delta D / \Delta f<1,1 \times 10^{-7} \mathrm{~Hz}^{-1}$ para monocamada de tiol, ${ }^{13}$ é indício de formação de filme rígido e homogêneo sobre o cristal de quartzo. Os valores de $\Delta D$ de diferentes harmônicos obtidos para o processo de formação das monocamadas de L-cisteína são muito pequenos com relação ao respectivo $\Delta f\left(-\Delta D / \Delta f<0,9 \times 10^{-7} \mathrm{~Hz}^{-1}\right.$, Figura $1 \mathrm{~S}$, material suplementar), evidenciando que a L-cisteína se adsorve rigidamente. Desta forma, é possível afirmar que $\theta(t)$ é proporcional a $\Delta f(\mathrm{t})$, e a Equação 6 se torna Equação 8:

$$
\Delta f(t)=\Delta f_{\text {máx }}\left[1-e^{(-\tau t)}\right]
$$

sendo que $\Delta f_{\text {máx }}$ representa a máxima adsorção de adsorbato no equilíbrio, para uma determinada concentração.

Realizando o ajuste dos dados experimentais das curvas de $-\Delta f(\mathrm{t})^{12}$ a partir do início do processo de adsorção até o início do processo de lavagem do cristal de quartzo (entre os pontos B a D mostrados na Figura 1) obtêm-se $\tau$. É mostrado, na Figura 2, exemplo do ajuste dos dados experimentais com a Equação 8. Os resultados dos ajustes estão resumidos na Tabela 1 e mostrados em gráfico na Figura 3.

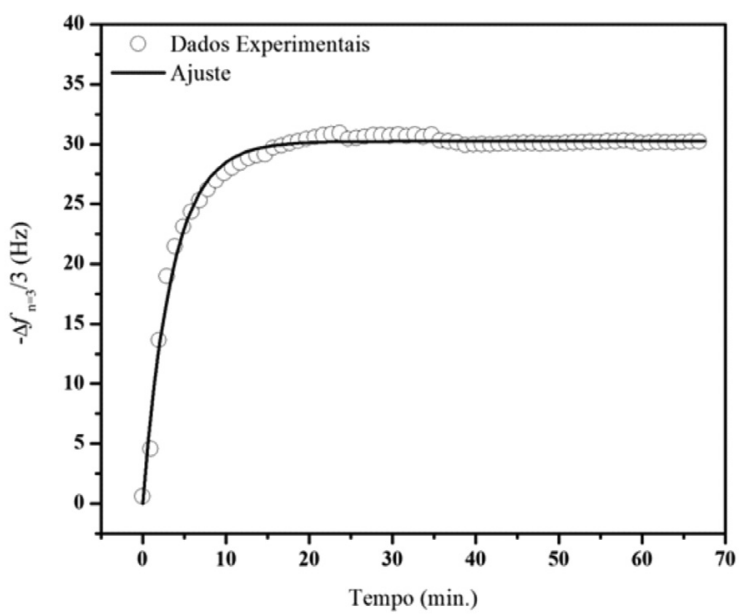

Figura 2. Exemplo de ajuste de dados experimentais com a Equação $8\left(R^{2}\right.$ = 97\%). Os dados experimentais são do processo de adsorção de L-cisteína a partir de solução aquosa de concentração $0,10 \times 10^{-4} \mathrm{M}$. O período de estabilização foi omitido

Desde que $\tau=k_{a} C+k_{d}$, um gráfico de $\tau$ vs $C$ para a série de concentração de adsorção de tiol fornece uma reta com coeficiente angular de $k_{a}$ e linear de $k_{d}$ (Figura 3 ). Os valores encontrados de $k_{d} \mathrm{e}$ $k_{a}$ pela regressão linear da melhor curva $\left(\mathrm{R}^{2}=97 \%\right)$ são $(4,2 \pm 0,4) \mathrm{x}$ $10^{-3} \mathrm{~s}^{-1}$ e $75 \pm 6 \mathrm{M}^{-1} \mathrm{~s}^{-1}$, respectivamente. Importante notar que $k_{d}>0$, ou seja, o processo de formação da camada de L-cisteína em ouro é um processo em equilíbrio, significando que, em qualquer momento durante o processo de formação da monocamada, ocorre processo de dessorção de moléculas de tiol sobre a superfície de ouro.

A partir dos resultados de $k_{a}$ e $k_{d}$ é possível determinar a constante de equilíbrio $\left(K_{e q}\right)$ do processo de adsorção da L-cisteína no ouro (Equação 9):

$$
K_{e q}=\frac{k_{a}}{k_{d}}
$$


Tabela 1. Valores de $\tau\left(10^{-3} \mathrm{~s}^{-1}\right)$ obtidos dos ajustes dos dados experimentais do processo de adsorção de L-cisteína em ouro a partir de diferentes concentrações aquosas desse adsorbato

\begin{tabular}{cc}
\hline Concentração de L-cisteína $\left(10^{-4} \mathrm{M}\right)$ & $\tau\left(10^{-3} \mathrm{~s}^{-1}\right)$ \\
\hline 0,1 & $5 \pm 1$ \\
0,5 & $12 \pm 3$ \\
1,0 & $13 \pm 4$ \\
2,5 & $22 \pm 7$ \\
5,0 & $41 \pm 4$ \\
\hline
\end{tabular}

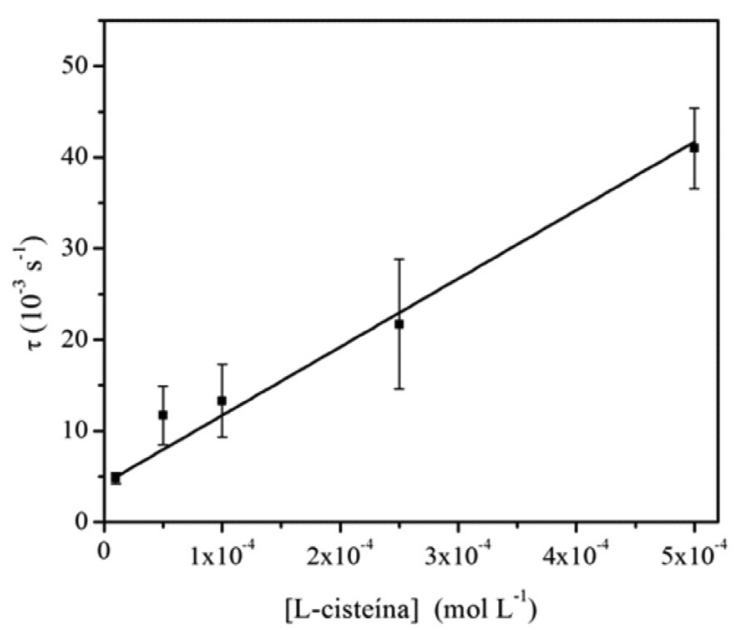

Figura 3. Dependência de $\tau$ com a concentração de L-cisteína utilizada. A regressão linear dos dados possui coeficiente linear $k_{d}$ e angular $k_{a}$

Utilizando a relação, encontra-se $K_{e q}=(1,8 \pm 0,3) \times 10^{4} \mathrm{M}^{-1}$.

A energia livre de adsorção da L-cisteína em ouro pode ser obtida a partir da constante de equilíbrio (Equação 10):

$$
\Delta G_{a d s}=-R T \ln K_{e q}
$$

Por meio da Equação 10, encontra-se $\Delta \mathrm{G}_{a d s}=-(5,8 \pm 0,2) \mathrm{kcal}$ $\mathrm{mol}^{-1}$.

Importante notar que esse valor se aproxima dos teóricos ${ }^{3,9}$ $\left(-5 \mathrm{kcal} \mathrm{mol}^{-1}\right)$ e daqueles encontrados experimentalmente para outros tióis reportados na literatura. ${ }^{12,26}$ Além do mais, a similaridade desse valor com aquele encontrado por Blomgren et al. ${ }^{27}$ para a adsorção de 1-butanotiol em mercúrio, de -4,9 $\mathrm{kcal} \mathrm{mol}^{-1}$, evidencia que a maior contribuição para a adsorção de tiol é a formação de tiolato.

Embora existam indícios de forte sistema organizacional das moléculas de L-cisteína adsorvidas em ouro, ${ }^{28}$ não é possível estimar exatamente qual a contribuição dessas interações para a energia livre de adsorção. O valor obtido pela técnica de QCM está relacionado com a energia livre do processo de adsorção, ou seja, formação do tiolato, sem a contribuição de processos organizacionais.

\section{CONCLUSÕES}

Foi observado que a adsorção de L-cisteína em ouro a partir de solução aquosa nas concentrações utilizadas é um processo rápido e obedece a isoterma de Langmuir. A partir dos resultados, foi possível demonstrar que o sistema estudado é um equilíbrio químico entre sítios livres de ouro e L-cisteína em solução. Os parâmetros cinéticos e termodinâmicos calculados foram $k_{d}=(4,2 \pm 0,4) \times 10^{-3} \mathrm{~s}^{-1}, k_{a}=75$ $\pm 6 \mathrm{M}^{-1} \mathrm{~s}^{-1}, K_{e q}=(1,8 \pm 0,3) \times 10^{4} \mathrm{M}^{1}$ e $\Delta G_{a d s}=-(5,8 \pm 0,2) \mathrm{kcal} \mathrm{mol}^{-1}$, sendo que o valor de $\Delta G_{a d s}$ é semelhante à energia livre de adsorção de outros alconatióis em ouro.

\section{MATERIAL SUPLEMENTAR.}

O material suplementar (Figura 1S) está disponível gratuitamente em http://quimicanova.sbq.org.br, no formato PDF. São mostrados, nesta figura, os harmônicos de frequência de oscilação do cristal de quartzo e os parâmetros de dissipação correspondentes $(n=3$ e $n=$ 5) obtidos durante o processo de adsorção de L-cisteína em ouro a partir de diferentes concentrações aquosas desse adsorbato.

\section{AGRADECIMENTOS}

À FAPESP, CAPES e CNPq pelo suporte financeiro.

\section{REFERÊNCIAS}

1. Bain, C. D.; Troughton, E. B.; Tao, Y. T.; Evall, J.; Whitesides, G. M.; Nuzzo, R. G.; J. Am. Chem. Soc. 1989, 111, 321.

2. Bain, C. D.; Biebuyck, H. A.; Whitesides, G. M.; Langmuir 1989, 5, 723.

3. Pradeep, T.; Nano: the essentials. Understanding nanoscience and nanotechnology, Tata McGraw-Hill: New Delhi, 2007.

4. Wink, T.; Zuilen, S. J. V.; Bult, A.; Bennekom, W. P. V.; Analyst 1997, 122, 43R

5. Love, J. C.; Estroff, L. A.; Kriebel, J. K.; Nuzzo, R. G.; Whitesides, G. M.; Chem. Rev. 2005, 105, 1103.

6. Pesquero, N. C.; Pedroso, M. M.; Watanabe, A. M.; Goldman, M. H. S.; Faria, R. C.; Roque-Barreira, M. C.; Bueno, P. R.; Biosens. Bioelectron. 2010, 26, 36 .

7. Briand, E.; Salmain, M.; Compère, C.; Pradier, C-M.; Colloids Surf., $B$ 2006, 53, 215.

8. Pedroso, M. M.; Watanabe, A. M.; Roque-Barreira, M. C.; Bueno, P. R.; Faria, R. C.; Microchem. J. 2008, 89, 153.

9. Ulman, A.; Chem. Rev. 1996, 96, 1533.

10. Hong, H-G.; Park, W.; Electrochim. Acta 2005, 51, 579.

11. Mccarley, R. L.; Dunaway, D. J.; Willicut, R. J.; Langmuir 1993, 9 , 2775 .

12. Karpovich, D. S.; Blanchard, G. J.; Langmuir 1994, 10, 3315.

13. Campiña, J. M.; Souza, H. K. S.; Borges, J.; Martinsa, A.; Gonçalves, M. P.; Silva, F.; Electrochim. Acta 2010, 55, 8779.

14. Love, J. C.; Wolfe, D. B.; Haasch, R.; Chabinyc, M. L.; Paul, K. E.; Whitesides, G. M.; Nuzzo, R. G.; J. Am. Chem. Soc. 2003, 125, 2597.

15. Yang, W.; Gooding, J. J.; Hibbert, D. B.; J. Electroanal. Chem. 2001, $516,10$.

16. Hernández, P.; Vicente, J.; Hernández, L.; Electroanalysis 2003, 15 , 1625 .

17. Paulo, T. D. F.; Diógenes, I. C. N.; Abruña, H. D.; Langmuir 2011, 27, 2052.

18. Kim, Y. S.; Yi, S. Y.; Kim, J.; Kim, M.; Kim, C-S.; Chung, B. H.; Kim, J. S.; Biosens. Bioelectron. 2009, 25, 248.

19. Qingwen, L.; Hong, G.; Yiming, W.; Guoan, L.; Jie, M.; Electroanalysis 2001, 13, 1342 .

20. Ihs, A.; Liedberg, B.; J. Colloid Interface Sci. 1991, 144, 282.

21. Arrigan, D. W. M.; Bihan, L. L.; Analyst 1999, 124, 1645.

22. Fatibello-Filho, O.; Capelato, M. D.; Quim. Nova 1992, 15, 28.

23. Damos, F. S.; Mendes, R. K.; Kubota, L. T.; Quim. Nova 2004, 27, 970.

24. Cooper, M. A.; Singleton, V. T.; J. Mol. Recognit. 2007, 20, 154.

25. Sauerbrey, G. Z.; Z. Phys. 1959, 115, 206.

26. Zuo, G.; Liu, X.; Yang, J.; Li, X.; Lu, X.; J. Electroanal. Chem. 2007, $605,81$.

27. Blomgren, E.; Bockris, J. O. M.; Jesch, C.; J. Phys. Chem. 1961, 65, 2000.

28. Zhang, J.; Chi, Q.; Nielsen, J. U.; Friis, E. P.; Andersen, J. E. T.; Ulstrup, J.; Langmuir 2000, 16, 7229. 


\section{DETERMINAÇÃO DOS PARÂMETROS CINÉTICOS E TERMODINÂMICOS DA ADSORÇÃO DE L-CISTEÍNA EM OURO POR MEIO DA TÉCNICA DE MICROBALANÇA A CRISTAL DE QUARTZO}

Adriano dos Santos, Josiel José da Silva e Paulo Roberto Bueno*

Instituto de Química, Universidade Estadual Paulista, Rua Francisco Degni, 55, 14800-900 Araraquara - SP, Brasil

Ailton Massaiti Watanabe

Faculdade de Filosofia, Ciências e Letras de Ribeirão Preto, Universidade de São Paulo, Av. Bandeirantes, 3900, 14040-901 Ribeirão Preto - SP, Brasil
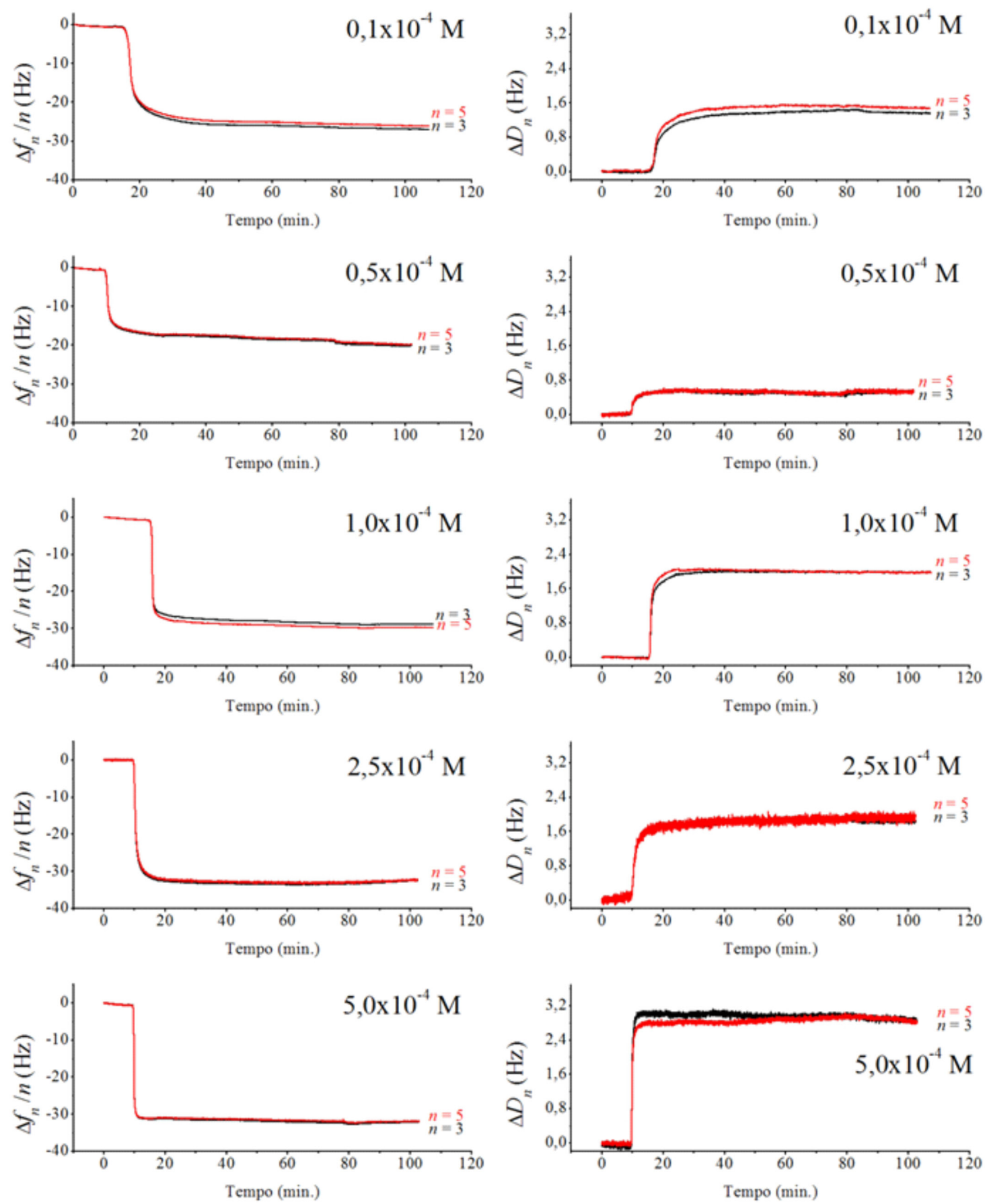

Figura 1S. Harmônicos de frequência de oscilação do cristal de quartzo e os parâmetros de dissipação correspondentes, $n=3(-)$ e $n=5(-)$, obtidos durante o processo de adsorção de L-cisteína em ouro a partir de diferentes concentraçães aquosas desse adsorbato 